\title{
REDUCTION THEORY IN ALGEBRAIC NUMBER FIELDS
}

\author{
BY HANS ZASSENHAUS
}

Communicated by Olga Taussky Todd, February 19, 1974

When is the half-group $\operatorname{GL}\left(n, Z^{\geqq 0}\right)$ of the unimodular matrices of degree $n$ over the half-ring $Z^{\geqq 0}$ of the nonnegative integers finitely generated ? ${ }^{1}$ Precisely if $n<3$.

Here the reduction of finite real extensions $E$ of the rational number field is based on Theorem 1 stating the finiteness of the number of all matrices of degree $n$ over $\boldsymbol{Z}^{\geqq 0}$ with a given irreducible characteristic polynomial over $\boldsymbol{Z}$, the rational integer ring, and on the following generalization of a well-known Frobenius theorem (Theorem 2): Let the semisimple commutative hypercomplex system $A$ over $\boldsymbol{R}$, the real number field, contain a semiring $H$ that is closed for the natural topology of $A$ such that $A=H+(-H), H \cap-H=\{0\}$ (pointed cone semiring). Then there are finitely many $\boldsymbol{R}$-homomorphisms $\theta_{i}(1 \leqq i \leqq s)$ of $A$ into the complex number field $C$ such that (1) $\bigcap_{i=1}^{s} \operatorname{ker} \theta_{i}=0$, (2) $\operatorname{ker} \theta_{i}+\operatorname{ker} \theta_{k}=$ $A$ ( $1 \leqq i<k \leqq s)$, (3) $A \theta_{i}=\boldsymbol{R}(1 \leqq i \leqq \rho ; 0<\rho \leqq s), \rho$ maximum, (4) for each $\rho$-tuple of nonnegative real numbers $\alpha_{1}, \cdots, \alpha_{\rho}$ there is an element $h$ of $H$ for which $h \theta_{i}=\alpha_{i}(1 \leqq i \leqq \rho)$, and (5) the set $C=\left\{\left(h \theta_{1}, \cdots, h \theta_{s}\right) \mid h \in\right.$ $\left.H \& 0 \leqq\left|h \theta_{\imath}\right| \leqq 1(1 \leqq i \leqq s)\right\}$ is a closed convex subset of $C^{1 \times s}$ containing 0 and closed under multiplication, and conversely. Note that $\left|\lambda_{i}\right| \leqq$ $\max _{1 \leqq j \leqq \rho}\left|\lambda_{j}\right|(1 \leqq i \leqq s)$ for $\left(\lambda_{1}, \cdots, \lambda_{s}\right)$ of $C$.

Theorem 1 is applied to a dedekind module $M$ of $E$ that is invariant under the $E$-order $\Lambda$. Any basis of $M$ over $Z$ leading to an irreducible integral representation $\Delta$ of $\Lambda$ representing a given primitive element $\omega$ of $E$ contained in $\Lambda$ by an integral matrix $\Omega$ of degree $n$ over $Z^{\geqq 0}$ permits the repeated formation of certain $\alpha \beta$-successors (predecessors) defined as

$$
S_{\alpha \beta}^{\varepsilon}(\Omega)=T_{\alpha \beta}^{-\varepsilon} \Omega T_{\alpha \beta}^{\varepsilon}
$$

$\left(\alpha \neq \beta, 1 \leqq \alpha \leqq n, 1 \leqq \beta \leqq n, \varepsilon= \pm 1, S_{\alpha \beta}^{\varepsilon}(\Omega) \in\left(Z^{\geqq 0}\right)^{n \times n}\right)$ defining an oriented finite graph $\bar{\Gamma}(\Omega)$ with a finitely presented fundamental group generated

AMS (MOS) subject classifications (1970). Primary 12A45, $12 \mathrm{~A} 50$.

1 This question was raised recently by G. Pall; it started the present exploration of a semigroup theoretic generalization of Lagrange's reduction theory. We utilize the subsemigroup $S_{n}$ of $\mathrm{GL}(n, Z \geqq 0)$ which is generated by the permutation matrices and the transvection matrices $T_{\alpha \beta}=I_{n}^{+}\left(\delta_{i \alpha} \delta_{k \beta}\right)(\alpha \neq \beta, 1 \leqq \alpha \leqq n, 1 \leqq \beta \leqq n)$ which is proper precisely if $n \geqq 3$. 
by fundamental loops corresponding to finitely many integral matrices commuting with $\Omega$ and generating a subgroup $U_{\Omega}$ of the image of the unit group, $U(\Lambda)$, of $\Lambda$ under $\Delta$. An estimate based on Theorem 2 and the geometry of numbers is given such that $U_{\Omega^{v}}\left\langle-I_{n}\right\rangle=U(\Lambda) \Delta$ if $v \geqq v_{0}$. A method for obtaining a representative set of the ideal classes of $\Lambda$ is developed in analogy to the method using continued fractions for real quadratic number field arithmetics.

A dualization method giving a new interpretation of the basic paper on 'matrix classes corresponding to an ideal and its inverse' (Illinois J. Math. 1 (1957), 108-113) by Olga Taussky is used in the course of the constructions.

Department of Mathematics, Ohio State University, Columbus, Ohio 43210 